Artigo / Article

\title{
Tratamento de suporte e quelação de ferro em pacientes com síndromes mielodisplásicas
}

\author{
Supportive care, tranfusion and chelation therapy for patients with myelodysplastic syndromes
}

Elizabeth X. Souto

\section{Introdução}

As síndromes mielodisplásicas (SMD) são um grupo heterogêneo de distúrbios hematológicos que ocorrem mais comumente em idosos, freqüentemente associado a anemia crônica e dependência transfusional. Cerca de $80 \%$ a $90 \%$ dos pacientes com SMD são dependentes de tranfusões; desta forma, a sobrecarga de ferro é inevitável e pode levar a danos teciduais graves.

O tratamento de suporte hemoterápico, para manter níveis de hemoglobina adequados para a oxigenação tecidual, bem como a quelação de ferro, quando indicada, são condutas fundamentais no acompanhamento de pacientes com SMD.

\section{Tratamento de suporte}

O objetivo do tratamento de suporte é reduzir a morbimortalidade relacionada a hematopoese ineficaz e melhorar a qualidade de vida dos pacientes com SMD. É utilizado principalmente para os pacientes que não respondem aos fatores estimuladores de colônia (Eritropoetina e/ou G-CSF) ou a outros tratamentos, e mantêm-se dependentes de transfusões de hemoderivados.

Hospital Brigadeiro, coordenadora do Ambulatório de Síndromes Mielodisplásicas e Mieloproliferativas Crônicas Laboratório Diagnósticos da América, médica da Hematologia e Citometria de Fluxo

Correspondência: Elizabeth Xisto Souto

Rua Cotoxó, 637 - apto 171

05021-000 - Perdizes - São Paulo - SP - Brasil

E-mail: xistosan@terra.com.br 
O nível de hemoglobina adequado varia de acordo com características específicas de cada paciente, como idade, comorbidades, estilo de vida e aspectos profissionais. ${ }^{1}$ Os sintomas de anemia são mais importantes que o nível de hemoglobina isoladamente, porém, há recomendações para que as transfusões sejam sempre indicadas em casos com hemoglobina abaixo ou iagual a 8,0 g\%. ${ }^{2}$ Além disso, deve se considerar que nível ótimo de hemoglobina seria acima ou igual a 10 g\%, evitando-se, desta forma, os danos cardíacos relacionados a anemia crônica.

Alguns autores sugerem o uso de concentrado de hemácias leucodepletados e, em pacientes candidatos a transplante de medula óssea, hemoderivados de doadores CMV negativos. ${ }^{2}$

A transfusão de concentrados de plaquetas está indicada em pacientes com contagem de plaquetas inferior a $20.000 / \mathrm{mm}^{3}$ e que apresentem febre e/ou fenômenos hemorrágicos e/ou que estejam na vigência de tratamento agressivo.

Além de transfusões de hemoderivados (concentrado de hemácias e de plaquetas), o tratamento de suporte é constituído também de profilaxia e tratamento de infecções de repetição, através do uso de fatores estimuladores de colônia (G-CSF) e quando necessário antibioticoterapia.

A neutropenia pode ser melhorada com a utilização de fator estimulador de colônia de granulócitos (G-CSF) e, se houver febre, está indicada antibioticoterapia empírica de amplo espectro. Não há evidências convincentes de que se deva utilizar antibioticoterapia profilática de rotina.

\section{Sobrecarga de ferro}

A sobrecarga de ferro em pacientes que recebem transfusões freqüentes é invitável, uma vez que uma unidade de concentrado de hemácias tem em média 200 mg a 250 mg de ferro e a perda natural diária de ferro é de $1 \mathrm{mg}$ a $2 \mathrm{mg}$ (Figura1).

Portanto, um paciente que recebe duas unidades de concentrado de hemácias por mês, receberá 24 unidades por ano, ou seja de 4,8 a 6,0 g de ferro em um ano, mais que o nível de ferro corporal normal que é de 3 a $4 \mathrm{~g}$. Em quatro anos receberá cerca de 20 a 25 g de ferro, níveis em que ocorrem as seqüelas relacionadas a sobrecarga de ferro.

Um recente estudo demonstrou que a dependência transfusional em pacientes com SMD está relacionada a pior sobrevida. Entretanto, isto pode ser devido a outros fatores que não a sobrecarga de ferro isoladamente, como, por exemplo, a piora da falência de medula óssea, levando a infecções e complicações hemorrágicas. ${ }^{3}$ As principais complicações relacionadas a sobrecarga de ferro são cardíacas, hepáticas e endócrinas, porém não há estudos comparando a freqüência destas complicações em pacientes com SMD e sobrecarga de ferro e casos-controle da mesma faixa etária. Desta forma, não está claro se estas complicações são mais

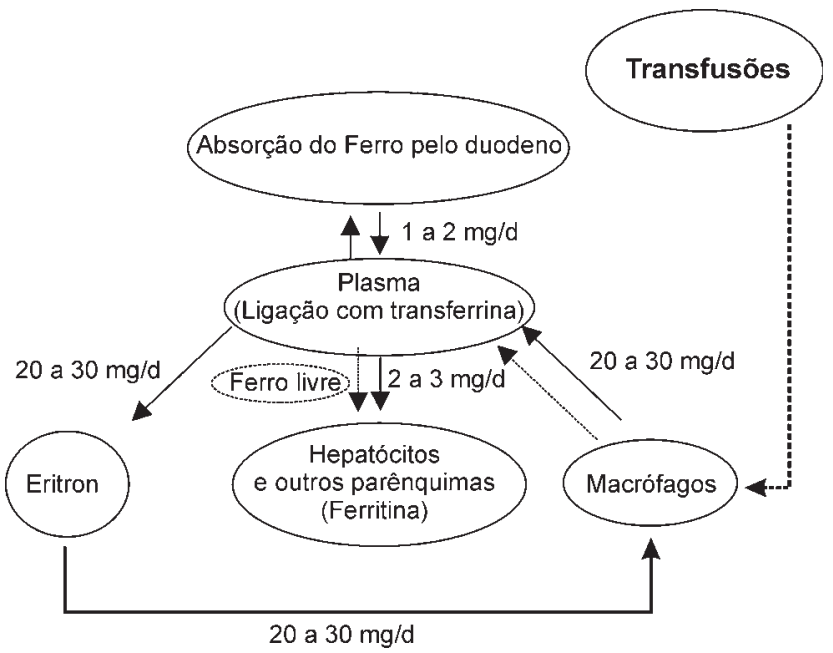

Figura 1. Modificado de John Porter, Hematology/Oncology Clinics, vol19, sup1, 2005

comuns em pacientes com SMD do que na população de idosos normais.

Um estudo incluindo 46 pacientes com SMD que receberam pelo menos 50 unidades de concentrado de hemácias (variação 50-150, mediana de 79). ${ }^{4}$ Vinte desenvolveram sinais de sobrecarga de ferro e insuficiência cardíaca, acompanhada por arritimias em alguns casos. Doze pacientes apresentaram lesão hepática e cinco desenvolveram diabetes melito. Insuficiência cardíaca congestiva grave foi a causa de óbito em 14 pacientes, nenhum paciente foi a óbito por complicações hepáticas. Os dados sugerem que as alterações cardíacas são as mais freqüentes e graves complicações em pacientes com SMD.

Não está totalmente esclarecido o quanto a sobrecarga de ferro é responsável por estas complicações cardíacas, uma vez que outros fatores possam estar envolvidos, como os efeitos da anemia crônica, doenças concomitantes, complicações da falência de medula óssea ou o processo de envelhecimento.

Por outro lado, a anemia crônica leva a um aumento da função cardíaca para melhorar a oxigenação tecidual, o que pode pode causar miocardiopatia dilatada, aumento da contratilidade do ventrículo esquerdo e gradual deterioração da reserva contrátil. Desta forma, um miocárdio já insuficiente poderia ser mais susceptível aos efeitos da sobrecarga de ferro.

\section{Avaliação da sobrecarga de ferro}

A estimativa do ferro corporal total e da distribuição do ferro nos tecidos é fundamental para o diagnóstico da sobrecarga de ferro e para o monitoramento em tratamentos de quelação.

A avaliação dos níveis de ferritina sérica é usada com freqüência para avaliação de sobrecarga de ferro. Apesar de 
haver uma boa correlação de elevados níveis com aumento do risco de óbito por doença cardíaca, é sabido que os seus níveis são influenciados por condições como infecção, quadros inflamatórios e deficiência de ascorbato. Este método é bastante útil no monitoramento de resposta a tratamento de quelação de ferro, porém não na avaliação do nível de ferro corporal total.

O fígado é o orgão com maior estoque de ferro, a avaliação da quantidade de ferro hepático (LIC - liver iron content), pode ser feita por biópsia hepática e predizer a quantidade do ferro corporal total através de cálculos. Entretanto, é uma técnica invasiva e que está contra-indicada em boa parte dos pacientes com SMD devido a plaquetopenia.

A abordagem não invasiva através de ressonância magnética $\mathrm{T} 2 *$ pode ser utilizada com boa acurácia para mensurar a quantidade de ferro hepático e calcular o ferro corporal total, porém, nem sempre é de fácil acesso.

A avaliação da quantidade de ferro hepático por SQUID (superconducting quantum interference device) também apresenta excelente correlação com a quantidade de ferro hepático avaliado por biópsia, porém é um método de alto custo e de difícil acesso, disponível em poucos serviços no mundo. ${ }^{5}$

\section{Tratamento da sobrecarga de ferro}

\section{Quelantes de ferro disponíveis}

\section{Deferoxamine}

É um quelante hexadentado com uma alta afinidade e seletividade pelo ferro, uma molécula de deferoxamine se liga a um átomo de ferro, formando um complexo estável que é excretado pela bile e pela urina.

Utilizado clinicamente há cerca de 35 anos, é altamente efetivo na profilaxia e tratamento da sobrecarga de ferro. Há poucos estudos em pacientes com SMD, porém há dados que sugerem que o tratamento com desferoxamine pode melhorar a hematopoese, com diminuição da necessidade transfusional e melhora da plaquetopenia e da neutropenia em alguns pacientes, muito embora os mecanismos não estejam bem esclarecidos. ${ }^{6}$

As limitações da terapia com deferoxamine estão relacionadas à sua pobre biodisponibilidade oral e curta meiavida plasmática; portanto, sua infusão deve ser lenta, de 8 a 12 horas por dia e deve ser utilizado de cinco a sete dias por semana para que haja boa efetividade.

Além do desconforto para o paciente, devido à longa duração de infusão e reações cutâneas no local da punção, o equipamento utilizado (bomba de infusão contínua), não está amplamente disponível em muitos países. Estes fatores levam, muitas vezes, à dificuldade de instituição do tratamento e de adesão do paciente ao tratamento.

No Brasil, muitas instituições apresentam também dificuldade de aquisição da droga para tratamento de pacientes com SMD.
Doses recomendadas: $20 \mathrm{mg}$ a $40 \mathrm{mg} / \mathrm{kg} / \mathrm{dia}$, infusão SC ou EV, 8 a 12 hs por dia, 5 a 7 dias por semana.

\section{Deferiprona}

É um quelante bidentado, ativo na forma oral, tem alta taxa de absorção intestinal, e todo o ferro quelado é eliminado pela urina. Tem capacidade de ligação ao ferro menor que o deferoxamine, uma vez que são necessárias três moléculas de deferiprona para se ligar a uma de ferro. Utilizado na forma oral, três vezes ao dia.

Desenvolvido há cerca de vinte anos, é um quelante oral aprovado na Europa como segunda linha de tratamento para pacientes intolerantes ao tratamento com deferoxamine ou com resposta insatisfatória ao mesmo. Não foi aprovado nos Estados Unidos pelo Food and Drugs Administration.

Estudos mostram que é mais efetivo que o deferoxamine na profilaxia da sobrecarga de ferro cardíaca. Apresenta como efeitos adversos mais relevantes a neutropenia e a agranulocitose em cerca de $8,5 \%$ e $0,5 \%$ dos pacientes, respectivamente .

Há poucos estudos com o uso de deferiprona em pacientes com SMD; um estudo piloto interrompido precocemente pelo risco de agranulocitose e outro com pacientes não talassêmicos, incluindo 18 pacientes com SMD, um desenvolveu agranulocitose e dois, discreta diminuição do número de plaquetas. ${ }^{7,8}$

\section{Deferasirox}

É um novo quelante tridentado, ativo na forma oral, requerendo duas moléculas para formar um complexo estável com cada átomo de ferro, a eliminação do ferro quelado é principalmente pela bile. Sua meia-vida plasmática é de 11 a 19 horas, é administrado oralmente, uma vez ao dia.

Estudos sugerem que o deferasirox é efetivo no controle dos níveis de ferro na dose de $20 \mathrm{mg} / \mathrm{kg} / \mathrm{d}$. Estudos clínicos fase II e III em pacientes adultos e pediátricos com anemia dependente de transfusão, como beta talassemia, SMD e Anemia de Blackfan-Diamond, têm mostrado que o deferasirox é efetivo e apresenta boa tolerabilidade. ${ }^{9} \mathrm{Um}$ recente estudo fase II em pacientes com anemias dependentes de transfusão, incluindo 47 pacientes com SMD e outras anemias (52 pacientes), demonstrou que doses de $10 \mathrm{mg} / \mathrm{kg} / \mathrm{d}$ foram suficientes para manter o balanço de ferro em $20 \mathrm{mg}$ a $30 \mathrm{mg} / \mathrm{kg} /$ dia para diminuir os níveis de ferro hepático. ${ }^{10}$

\section{Quelação de ferro em pacientes com SMD}

A quelação de ferro sabidamente pode melhorar a sobrevida e a qualidade de vida em pacientes com beta talassemia homozigota. Pode-se observar este efeito especialmente após os 15 anos de idade, ou seja, após cerca de dez anos de tratamento. Há um grande número de publicações, com um vasto conhecimento nesta área, porém não necessariamente este possa ser aplicado em pacientes com SMD. 
Uma vez que a SMD é predominantemente uma doença do idoso e que, portanto, a expectativa de vida não é longa, discute-se se esta sobrevida seria suficiente para justificar a terapia de quelação. Por outro lado, os pacientes idosos poderiam ser mais vulneráveis aos efeitos tóxicos do ferro e, portanto, a terapia de quelação deveria ser iniciada mais precocemente. Desta forma, é necessário identificar quais os pacientes que se beneficiariam da terapia de quelação através da análise de sobrevida.

A análise de sobrevida dos pacientes pode ser baseada na classificação para SMD da Organização Mundial da Saúde (OMS) e no International Prognostic Scoring System (IPSS).

Considerando-se a classificação OMS, os pacientes com subtipos AR e ARSA têm uma mediana de sobrevida em torno de cinco anos, na sua maioria são dependentes de transfusões e provavelmente desenvolvem sobrecarga de ferro, estes se benificiariam da terapia de quelação. Os subtipos CRDM e CRDMS apresentam mediana de sobrevida em torno de três anos, os pacientes freqüentemente são dependentes de transfusão e podem desenvolver sobrecarga de ferro. Porém apresentam maior risco de transformação leucêmica e podem ter evolução bastante heterogênea. Pacientes com Síndrome do 5q- são potenciais candidatos a quelação de ferro, por apresentarem dependência transfusional e longa sobrevida. Os subtipos com excesso de blastos (AREB I e AREB II) apresentam sobrevidas curtas e não se justifica a terapia de quelação.

Utilizando-se o IPSS observam-se dois grupos que se benificiariam da quelação de ferro, os com escore de baixo risco (alguns pacientes com CRDM e CRDMS, a maioria dos pacientes AR e ARSA) com sobrevida em torno de cinco anos, e os com escore de risco intermediário I (alguns pacientes com CRDM e CRDMS), com sobrevida em torno de três anos. ${ }^{11}$

Recentemente, foi realizado um encontro em Nagasaki, Japão, com especialistas de diversos países para discussão sobre sobrecarga de ferro em SMD. Nele foram discutidos, além dos aspectos clínico-laboratoriais, as diferenças regionais e dificuldades das instituições em diagnóstico e tratamento; com base nesta discussão, foi elaborado um Consenso (Figura 2). ${ }^{12}$

\section{Sobrecarga de ferro em síndromes mielodisplásicas}

- As consequências clínicas da sobrecarga de ferro não tratada ou inadequadamente manuseada em pacientes com SMD são potencialmente complicações cardíacas, hepáticas e endócrinas.

- O objetivo da terapia de quelação em pacientes com SMD com sobrecarga de ferro é prevenir e tratar as complicações da mesma e melhorar a sobrevida.

- A terapia de quelação é altamente adequada e clinicamente importante em um subgrupo de pacientes com SMD,

Monitoramento da sobrecarga de ferro:

Quando se deve avaliar os estoques de ferro em pacientes com SMD?

- Ao diagnóstico e intervalos regulares, dependendo da freqüência das transfusões.

Quais os exames que devem ser utilizados para monitorar a sobrecarga de ferro?

- Ferritina sérica

- Saturação de transferrina

- Ressonância magnética de fígado

Com que frequência se deve monitorar a sobrecarga de ferro?

- Pelo menos a cada 3 meses em pacientes recebendo transfusões

Tratamento da sobrecarga de ferro

Quando deve ser considerado iniciar a quelação de ferro em pacientes com SMD?

- Quando o nível de ferritina sérica estiver de 1000 a $2000 \mathrm{ng} / \mathrm{ml}$, dependendo da frequência de transfusões

Quanto tempo a quelação deve continuar?

- Enquanto a terapia transfusional continuar e a sobrecarga de ferro for clinicamente relevante

Perfil dos pacientes:

Quais pacientes mais se beneficiam do tratamento da sobrecarga de ferro?

- Pacientes dependentes de transfusão

- Pacientes com níveis de ferritina > 1000 a $2000 \mathrm{ng} / \mathrm{ml}$ ou outra evidência de sobrecarga de ferro tecidual

- Pacientes com SMD baixo risco (IPSS baixo ou intermediário I, WHO: AR, ARSA, 5q-)

- Pacientes com SMD estável

- Pacientes livres de comorbidades que limitam o prognóstico e a sobrevida

- Candidatos a TMO

Figura 2. Sobrecarga de ferro em SMD, Consenso, Nagasaki, Japão, maio de 2005

Traduzido de Hematology/Oncology Clinics, 2005, vol 19, suppl 1, pag. 22 


\section{Considerações finais}

Recomendações e linhas gerais de tratamento de suporte e quelação de ferro em pacientes com SMD:

Tratamento de suporte:

- Transfusões de concentrado de hemácias $(\mathrm{CH})$ :

Transfundir sempre que hemoglobina $(\mathrm{Hb})=8,0 \mathrm{~g} \%$.

Transfundir se sinais e/ou sintomas de anemia, mesmo que Hb esteja $>8 g \%$.

Se possível manter $\mathrm{Hb}>10 \mathrm{~g} \%$.

Se possível transfundir $\mathrm{CH}$ leucodepletados.

Em pacientes candidatos a TMO transfundir $\mathrm{CH}$ de doador CMV negativo.

- Transfusões de plaquetas

Se plaquetas $<20.000 / \mathrm{mm}^{3}$ e/ou fenômenos hemorrágicos, febre, infecção, tratamento agressivo mieloablativo.

Não está indicado de rotina transfusões de plaquetas, em pacientes com plaquetas $=20.000 / \mathrm{mm}^{3}$ a menos que estejam presentes as condições citadas acima.

- Neutropenia

Se houver infecções de repetição está indicado o uso de fator estimulador de granulócito (G-CSF)

Se paciente é neutropênico ( $<1.000$ leucócitos $/ \mathrm{mm}^{3}$ ), com febre, sem foco infeccioso aparente, está indicada antibioticoterapia empírica de amplo espectro.

Se paciente neutropênico $\left(<1.000\right.$ leucócitos $\left./ \mathrm{mm}^{3}\right)$, com foco infeccioso identificado, está indicada antibioticoterapia empírica de amplo espectro além da cobertura específica de acordo com o foco.

A antibioticoterapia profilática não indicada de rotina

\section{Quelação de ferro}

Monitoramento da sobrecarga de ferro de ferro:

Quando se deve fazer o monitoramento da sobrecarga

- Ao diagnóstico e em intervalos regulares, dependendo da freqüência das transfusões.

Sugestão:

- Em pacientes que transfundem mensalmente, de 3/3 meses.

- Em pacientes que transfundem menos, exemplo a cada 2 meses, de 6/6meses. ramento:

Quais os exames que devem ser utilizados no monito-

- Ferritina (3/3meses).

- Saturação de transferrina (3/3meses).

- Ressonância magnética hepática (T2*), se possível (1 x por ano).

\section{Tratamento da sobrecarga de ferro:}

Quando se deve iniciar a quelação de ferro em pacientes com SMD?

- Quando os níveis de ferritina estiverem de 1000 a $2000 \mathrm{ng} / \mathrm{ml}$ e saturação de transferrina $>60 \%$ e dependendo da frequência das transfusões.

- Acima de 20 unidades de concentrado de hemácias transfundidas e presença das condições acima

Quanto tempo o tratamento de quelação de ferro deve continuar?

- Enquanto a terapia trasnfusional continuar e enquanto a sobrecarga de ferro for relevante.

Qual o perfil dos pacientes com SMD que se beneficiam da quelação de ferro?

- Pacientes dependentes de transfusão.

- Pacientes com ferritina de 1.000 a $2.000 \mathrm{ng} / \mathrm{ml}$ ou outra evidência de sobrecarga de ferro tecidual.

- Pacientes com mais de 20U de CH transfundidas.

- Pacientes com SMD dos seguintes subgrupos: Risco IPSS: baixo e intermediário I eWHO: AR, ARSA, alguns pacientes com CRDM e CRDMS e Del 5q-.

- SMD estável.

- Pacientes com SMD livres de comorbidade que limitem a sobrevida.

- Candidatos a TMO.

Qual deve ser o esquema utilizado para a quelação de ferro em pacientes com SMD?

- Deferoxamine 20 a $40 \mathrm{mg} / \mathrm{kg} /$ dia infusão contínua SC em bomba de infusão contínua, 5 a 7 dias por semana. Se ferritina $<2.000 \mathrm{ng} / \mathrm{ml}$, reduzir dose para no máximo $25 \mathrm{mg} / \mathrm{kg} / \mathrm{d}$

Recomendações:

- Orientações rigorosas quanto ao uso correto da bomba de infusão e assepsia do local de punção.

- Não utilizar em pacientes com plaquetas < 50.000/ $\mathrm{mm}^{3}$ pelo risco de hematomas nos locais de punção.

- Considerar no futuro a possibilidade do uso de novos quelantes orais em estudo.

\section{Abstract}

Myelodysplastic syndromes (MDSs) are a heterogeneous group of hematological disorders which are more common in the elderly and related to chronic anemia dependent on blood transfusions. Consequently, many of these patients develop iron overload which may lead to severe injury to tissues. Transfusions and chelation therapy, when indicated, are important for survival and to maintain the quality of life. Chelation therapy is indicated especially for MDS subtypes with a better prognosis and a sufficiently long survival to develop clinically relevant iron overload. Chelation therapy presents with some limitations in particular the long time required for deferoxamine infusion and the difficulties of patients to comply with 
treatment and to acquire an infusion pump. The clinical use of deferiprone, an oral chelator, is not indicated for MDS patients because of the risk of neutropenia and agranulocytosis. Deferasirox is a new oral chelator currently under clinical development that will probably be, in the future, an adequate option for MDS patients with iron overload. Additional studies in MDS patients are necessary to establish better diagnostic and chelation therapy criteria. Rev. bras. hematol. hemoter. 2006;28(3):204-209.

Key words: Myelodysplastic syndromes; supportive care; transfusion therapy; iron overload; chelation therapy.

\section{Referências Bibliográficas}

1. Murphy M F, Wallington TB, Kelsey P, Boulton F, Bruce M, Cohen $\mathrm{H}$, et al. Guidelines for the use of red cell transfusions. Br J Haematol 2001;113:24-31.

2. Alessandrino EP, Amadori S, Barosi G, Cazzola M, Grossi A, Liberato LN, et al. Evidence - and consensus - based practice guidelines for the therapy of primary mielodysplastic syndromes. A statement from the Italian Society of Hematology. Haematologica 2002; 87:1.286-1.306.

3. Cazzola M, Malcovati LN. Myelodysplastic Syndromes coping with ineffective hematopoiesis. N Engl J Med 2005 ; 352:536-8

4. Jaeger M, Aul C, Sohngen D, Germing U, Schneider W. Secondary hemochromatosis in polytransfused patients with myelodysplastic syndromes, Beitr Infusionsthe 1992;30:464-8.

5. Porter, JB. Monitoring and treatment of iron overload: State of the art and new approaches. Seminars in hematology 2005;42:(2), supl 1, S1-S21.
6. Jensen PD, Heickendorff L, Pedersen B, Bendix-Hansen K, Jensen FT, Christensen $\mathrm{T}$, et al. The effect of iron chelation on haemopoiesis in MDS patients with transfusional iron overload. Br J Haematol 1996;94:288-99.

7. Jaeger M, Aul C, Sohngen D, Germing U, Scheneider W. Iron overload in polytransfused patients with MDS: use of L1 for oral iron chelation. Drug of today 1992;28 (suppl A):143-7.

8. Kersten MJ, Lange R, Smeets ME, Vreugdenhil G, Roozendaal KJ, Lameijer $\mathrm{W}$, et al. Long-term treatment of transfusional iron overload with the oral iron chelator deferiprone (L1): a Dutch multicenter trial. Ann Hematol 1996;73:247-52.

9. Poter J, Vichinsky E, Rose C, Piga A, Olivieri N, Gattermann N, et al. Phase II study with ICL 670 (Exjade@), a once- daily oral iron chelator, in patients with various transfusion-dependent anemias and iron overload. Blood 2004;104 (Suppl11). Abstract 3193.

10. Gattermann N, Cazzola M, Greenberg P, Maertens J, Soulieres D, Rose C, et al. The efficacy and tolerability of ICL670, a oncedaily oral iron chelator, in patients with myelodisplastic syndromes (MDS) and iron overload. LeuK Res 2005;29(Suppl 1):S67.

11. Greenberg P, Cox C, LeBeau MM, Fenaux P, Morel P, Sanz G, et al. International scoring system for evaluating prognosis in myelodysplastic syndromes. Blood 1997;89:2079-88

12. Gattermann N, Porter J, Lopes LF, Seymour J. Iron overload in myelodysplastic syndromes. Hematology/oncology clinics 2005; 19(Suppl 1):18-25.

Avaliação: Editor e dois revisores externos.

Conflito de interesse: não declarado

Recebido: 09/03/2006

Aceito após modificações: 11/09/2006 